\title{
Paroxetine-induced hyponatremia
}

Paroxetine is a selective serotonin reuptake inhibitor (SSRI) used extensively in major depressive disorders, obsessivecompulsive disorder, panic disorder, and generalized anxiety disorder. In recent years, SSRIs have become the preferred agents in depression due to their relative safety and better tolerability. Gastrointestinal disturbances, central nervous system (CNS) side effects, and sexual dysfunction may be encountered as side effects during paroxetine therapy. Paroxetine is not known to interfere with any commonly performed laboratory assays. ${ }^{[1]}$ Hyponatremia is an underrecognized and potentially serious complication of paroxetine therapy, especially in older patients. ${ }^{[2]}$ Here we report a case of a 58-year-old woman with paroxetine-induced hyponatremia.

A 58-year-old married woman presented to the psychiatry OPD of our institute with two months' history of disturbance of sleep (early morning awakening), loss of interest in daily activities, and feelings of helplessness. She had a past history of thyrotoxicosis, which had been diagnosed in 1991 and for which she had been treated with carbimazole for four years. She had improved with the treatment and the drug was withdrawn gradually. On physical examination, her pulse was 86/min and BP was 140/80 $\mathrm{mm}$ of $\mathrm{Hg}$. No abnormality was detected on examination of the abdomen or the respiratory and cardiovascular systems. CNS examination revealed no focal neurological deficit. Mental status examination revealed a conscious, cooperative but retarded patient, with moderate depression and a stable mood with appropriate affect. Speech was low, with delayed reaction time. No formal thought disorder could be elicited and the higher cognitive functions were appropriately normal . Laboratory investigations ( $\mathrm{Hb} \%$, total leukocyte count, differential count, blood glucose level, blood urea and creatinine, T3, T4 and TSH estimation, and thyroid scan) were within normal limits. Considering the history of the patient and the mental status examination findings, a provisional diagnosis of moderate depressive disorder without somatic symptoms was considered. She was started on tablet paroxetine $12.5 \mathrm{mg}$ once daily for a week, which was then increased to $25 \mathrm{mg}$ at bedtime. She reported back to the psychiatry outpatient clinic after three weeks and some of her symptoms, including the sleep disturbance, had improved. After three months she was brought back to the psychiatry OPD with complaints of anorexia, weight loss, lassitude, and malaise. On physical examination she was mildly dehydrated with a pulse rate of 88/min and $\mathrm{BP}$ of 110/70 $\mathrm{mm}$ of $\mathrm{Hg}$. Examination of the abdomen and the respiratory and cardiovascular systems were within normal limits. CNS examination revealed no focal neurological deficit. Mental status examination revealed a conscious, cooperative patient with a depressive mood. No other psychopathology could be detected. Hb\%, total leucocyte count, differential count, blood glucose level, and blood urea and creatinine were within the normal range. Her serum sodium was $101 \mathrm{mEq} / \mathrm{l}$. Other electrolytes were within normal limits. The patient was admitted and treated with intravenous normal saline infusion and fluid restriction. Serial serum sodium measurements were done which were as follows: day 112 $\mathrm{mEq} / \mathrm{l}$ on day $4,126 \mathrm{mEq} / \mathrm{l}$ on day 10 , and $136 \mathrm{mEq} / \mathrm{l}$ on day 16. Her general condition improved and she was discharged with amitriptyline, a tricyclic antidepressant, $50 \mathrm{mg}$ at bedtime. She reported to the psychiatry OPD with significant improvement in her mental status two and four months after discharge from hospital. Her serum sodium levels were also within the normal range.

Hyponatremia is defined as a serum sodium concentration below $135 \mathrm{mEq} / \mathrm{l}{ }^{[3]}$ In this case, the patient developed severe hyponatremia $(101 \mathrm{mEq} / \mathrm{l})$. The correction of the patient's hyponatremia, combined with the substitution of paroxetine with amitriptyline, resulted in resolution of her symptoms, without recurrence of the hyponatremia. The fact that sodium returned to the normal range after discontinuing paroxetine supports medication-induced hyponatremia as the causative mechanism. The patient was not receiving any other medication or suffering from any systemic disease that might have contributed to the development of hyponatremia. Hyponatremia is a rare and potentially dangerous complication of SSRIs. ${ }^{[4,5]}$ SSRI-induced hyponatremia is common in the elderly agegroups ${ }^{[2]}$ but may also occur at younger ages. ${ }^{[6]}$ The other risk factors are female gender, smoking, low body weight, tumors, respiratory or CNS illness, previous history of hyponatremia, and other medications, particularly diuretics. ${ }^{[7]}$ In this patient some of these risk factors, i.e., female gender, elderly age (58 years), and depressive disorder, might have also contributed to the hyponatremia. SSRI-induced hyponatremia may have early onset (3-14 days) ${ }^{[8]}$ or late onset (3-5 months)..$^{\mid{ }^{|9|}}$ Though we did not monitor the plasma electrolytes in this patient after putting her on paroxetine, the patient reported with hyponatremia three months and three weeks after starting paroxetine. Syndrome of inappropriate secretion of anti-diuretic hormone, induced by paroxetine, is the probable mechanism for the development of hyponatremia. ${ }^{[2]}$

Physicians prescribing paroxetine, especially for elderly patients, should be alert to the possibility of hyponatremia presenting with subacute mental status changes. Patients may present with fatigue, weakness, and apathy, which may simulate worsening of depression. ${ }^{[10]}$ Periodic monitoring of serum electrolytes should be done, especially in patients at high risk. Milder cases (serum sodium 125-134 mEq/l) may be managed with discontinuation of SSRIs and fluid restriction, but more severe cases require aggressive medical management with hypertonic saline or normal saline and frusemide. ${ }^{[11]}$ 


\section{Ranjib Ghosh, Prasenjit Deka* D epartments of Pharmacology, *Psychiatry, Tripura Medical College and Dr. B.R. Ambedkar Teaching Hospital, Hapania, Tripura (W), India. E-mail: ghoshranjib@rediffmail.com}

\section{References}

1. Kelsey JE. Kaplin and Sadock's comprehensive textbook of psychiatry. $8^{\text {th }}$ ed. Sadock BJ, Sadock VA, editors. Lippincott Williams and Wilkins: Philadelphia; 2005.

2. Fabian TJ, Amico JA, Kroboth PD, Mulsant BH, Reynolds CF $3^{\text {rd }}$, Pollock BG Paroxetine induced hyponatremia in elderly due to the Syndrome of Inappropriate Secretion of Anti Diuretic Hormone (SIADH). J Geriator Psychiatry Neurol 2003;16:160-4.

3. Fourlanos $\mathrm{S}$, Greenberg P. Managing drug induced hyponatremia in adults. Aust Prescr 2003:26:114-7.
4. Romerrio SC, Radanowicz V, Schlienger RG. SIADH with epileptic seizures and coma in fluoxetine therapy (Article in German). Schweiz Rundsch Med Prax 2000;89:404-10.

5. Kirby D, Ames D. hyponatremia and serotonin reuptake inhibitors in elderly patients. Int J Geriatr Psychiatry 2001;16:484-93.

6. Corrington KA, Gatlin CC, Fields KB. A case of SSRI induced hyponatremia. J Am Board Fam Pract 2002;15:63-5.

7. Fonzo-Christe $\mathrm{C}$, Vogt $\mathrm{N}$. Susceptibility of the elderly patient to hyponatremia induced by Selective Serotonin Reuptake Inhibitors (Article in French). Therapie 2000;55:597-604.

8. Christe C, Vogt N. SSRI-induced SIADH in older people. J Am Geriator Soc 1999;47:630-1.

9. Flint AJ, Crosby J, Genik JL. Recurrent hyponatremia associated with fluoxetine and paroxetine. Am J Psychiatry 1996;153:134.

10. Leung VP, Chiu HF, Lam LC. Hyponatremia associated with Paroxetin. Pharmacopsychiatry 1998;31:32-4.

11. Bourgeois JA, Babine SE, Bahadur N. A case of SIADH and Hyponatremia associated with Citalopram. The Academy of Psychosomatic Medicine 2002. 